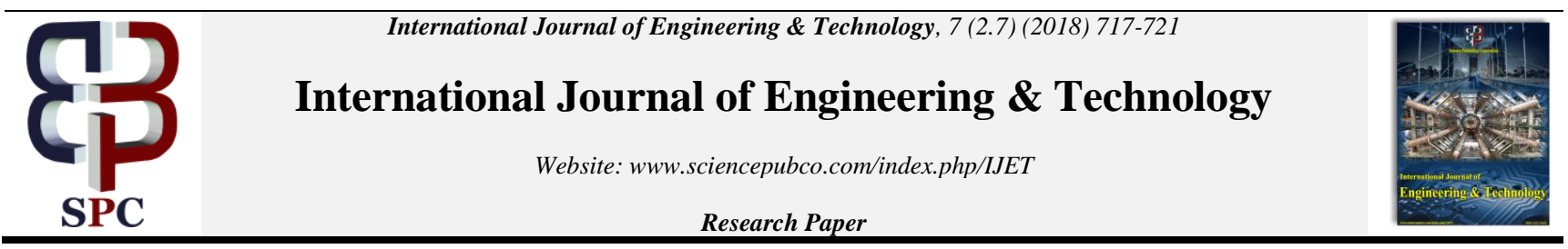

\title{
Classification of skin cancer images using TensorFlow and inception v3
}

\author{
Bhavya Sai $V^{1}$, Narasimha Rao G ${ }^{2}$, Ramya $\mathbf{M}^{3}$, Sujana Sree $\mathbf{Y}^{4}$, Anuradha $\mathbf{T}^{5}$ * \\ 1, 2,3,4,5 V R Siddhartha Engineering College, kanuru, Vijayawada, India. \\ *Corresponding author E-mail: atadiparty@gmail.com
}

\begin{abstract}
It is easy for a human eye to distinguish the images of similar appearance but classifying the images like that of cancer affected skin requires more expertise. And as the skin cancer cases are increasing globally, it requires more number of human experts. To overcome this problem, many people are working on constructing machine learning classifiers which can detect skin cancer automatically by classifying skin images. This paper concentrates on developing an approach for predicting skin cancer by classifying images using deep convolution neural network. The proposed work is tested on standard cancer dataset and obtained more than $85 \%$ accuracy.
\end{abstract}

Keywords: Classification; Deep Convolution Neural Network; Inception-V3; Machine Learning; Tensor Flow.

\section{Introduction}

\subsection{Motivation}

Today, almost every application in the world is directly or indirectly influenced by computer systems. Computers are used in diverse areas for various automated applications. In the diseases like cancer, identifying the disease at the early stage is very important for better treatment and cure. Today many people across the globe are suffering with skin diseases and number of skin cancer cases are more compared to any other types of cancer [1].As all the skin cancers are not the deadly cancers, by looking at the skin images, it is required to predict whether it is deadly cancer or not. From all skin cancers, melanoma cases are present in just 5\% of cases, but $75 \%$ of times, it may lead to death according to American Cancer Society [2,3].Detecting skin cancer by human doctors requires more expertise and knowledge and with increased number of skin cancer cases, it requires more number of experts who can correctly diagnose the disease. As it is difficult to get more number of human experts, today many researchers are working on developing machine learning algorithms to solve this problem. Automated detection of skin cancer is a challenging task [4] but by training the computer it can identify whether the skin is affected with deadly cancer or not. This paper proposes Deep learning approach to predict the cancer. The proposed work is implemented with Tensor Flow and Inception V3 models and tested on standard cancer dataset and obtained more than $85 \%$ accuracy.

\subsection{Problem Definition}

Skin cancer is the most common due to abnormal growth of skin cells. But this growth always may not be the symptom of deadly cancer. So, distinguishing correctly and proper treatment leads to better cure of the disease. In this paper, deep convolutional neural network (DCNN) [5], a machine learning classification technique is used to classify the skin cancer images. As accuracy is the most important factor in this problem, by taking more number of images for training the network and by increasing the number of iterations, the DCNN accuracy can be enhanced. Tensor Flow is a large scale machine learning system developed by Google [6] and Inception V3 is Google's CNN architecture [7].Here, the DCNN algorithm is implemented with Tensor Flow and Inception V3.

\subsection{Terminology}

\subsubsection{Types of Skin Cancer}

Skin cancer [8] affects people whose skin is highly exposed to the sun rays. Those who have lighter coloured skin have higher risk of cancer. There are two types of cancer. They are

Malignant Cancer In Fig.1 the cancer cells spread from one part of the body to the other there by even though, the cancer cells are removed from the initial affected part, it may raise from other parts. It is considered to be the deadly cancer. Sarcomas, Carcinomas [9] are the common varieties in this malignant cancer and melanoma though less in number is a serious type of cancer. 


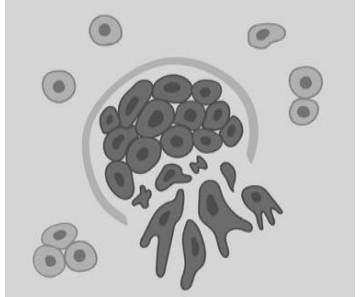

Fig. 1: Malign Cells

Benign Cancer In Fig.2 cells do not spread from one part of the body to the other part. It is called benign cancer. If the cancer cells are removed from initial place it will never come back again. It is regarded as normal cancer.

Most Common types of benign cancers are Adenomas, Meningioma's, Fibromas or fibroids, Papilloma's, lymphomas, Nevi, Myomas, Haemangioma, Neuromas, Osteochondromas [9].

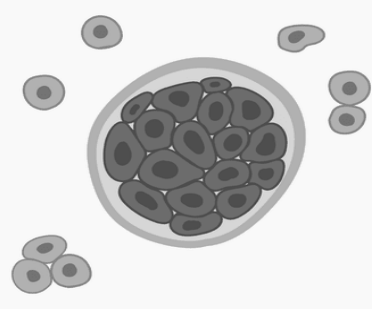

Fig. 2: Benign Cells

\subsection{Transfer learning}

Transfer learning is a machine learning technique [10] which is based on the concept of reusability.

Fig. 3 describes the inception V3 model which processes convolution, pooling, softmax and fully connected operations.

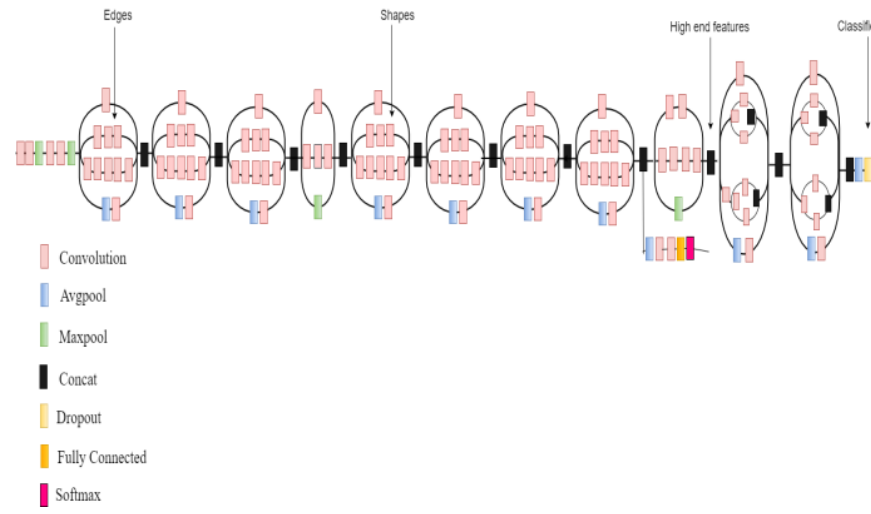

Fig. 3: Inception V3

Here a pre-trained neural network developed for one task can be used as the starting point of other. The image recognition model Inception-v3 contains two parts:

- Feature extraction part using convolutional neural network.

- Classification part using fully-connected and softmax layers.

The pre-trained Inception-v3 model succeeds in state-of-the-art accuracy for distinguishing universal things using 1000 classes, like "Zebra", "Computer", and "Dishes". The model extracts fea- tures from input images in the first part and classifies them based on those features in the second part [11].

\subsection{Tensor flow}

Tensor Flow is an artificial neural network having more than three layers as in Fig.4. It has one input, one output and multiple hidden layers [12].

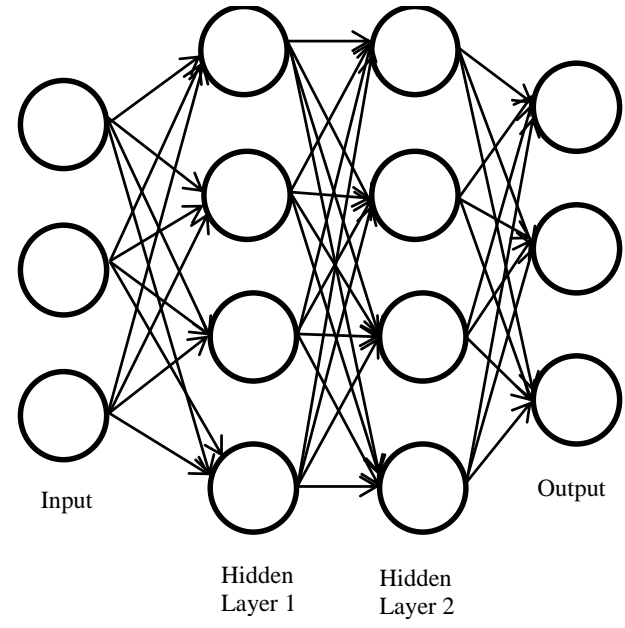

Fig. 4: Neural Network

It is a deep learning frame work developed by Google. It has control over every neuron (node) in the network and has libraries suitable for image processing. The weights of the neural network can be modified to achieve better performance [13].

\section{Literature Survey}

There are various techniques in image processing for image classification. Deep convolution neural network (DCNN) is a new approach for this problem. Researchers are using this technique recently in various classification problems. Deep learning models are used in many application areas of medicine $[14,15]$. DCNN is used to classify the time series signals in [16]. Here the time series signals are first converted to $2 \mathrm{D}$ images using recurrence plots and then $\mathrm{CNN}$ is used for image recognition. Early detection saves the life of many patients in cancer diseases and the detection of skin lesions is proposed in [17]. The proposed methodology was tested on ISIC dataset and found to be accurate. Skin cancer cases are increasing day by day and presently they are more in number compared to any other type of cancer US. By following VGG16, VGG19 \&Google Net models, deep convolution network was constructed and implemented on GPU architecture in [18]. The proposed approach obtained $73 \%$ accuracy. Classification of nonmalignant lesions from malignant was done in [19]. The training approach used deep residual networks with transfer learning. As the early symptoms of Melanoma cancer and moles are almost similar, it is very hard to distinguish both. RBF Gaussian SVM approach is used for classification in [20]. Classification and detection of skin cancer using 2D Wavelet decomposition and multilayer, radial basis neural networks is discussed in [21]. Usage of wavelet transforms and fuzzy logic for the skin cancer detection is discussed in [22]. Deeper models results in better skin cancer image classification [23]. Melanoma screening using segmentation before feature extraction is used in [24, 25]. 


\section{Architecture}

Fig. 5 shows the step by step process of the proposed work model $[26,27]$. The steps in the proposed classification model are as follows:

1. Perform convolution and pooling on the image recursively

2. Apply drop out and fully connected. Now the image must be classified according to the training class labels.

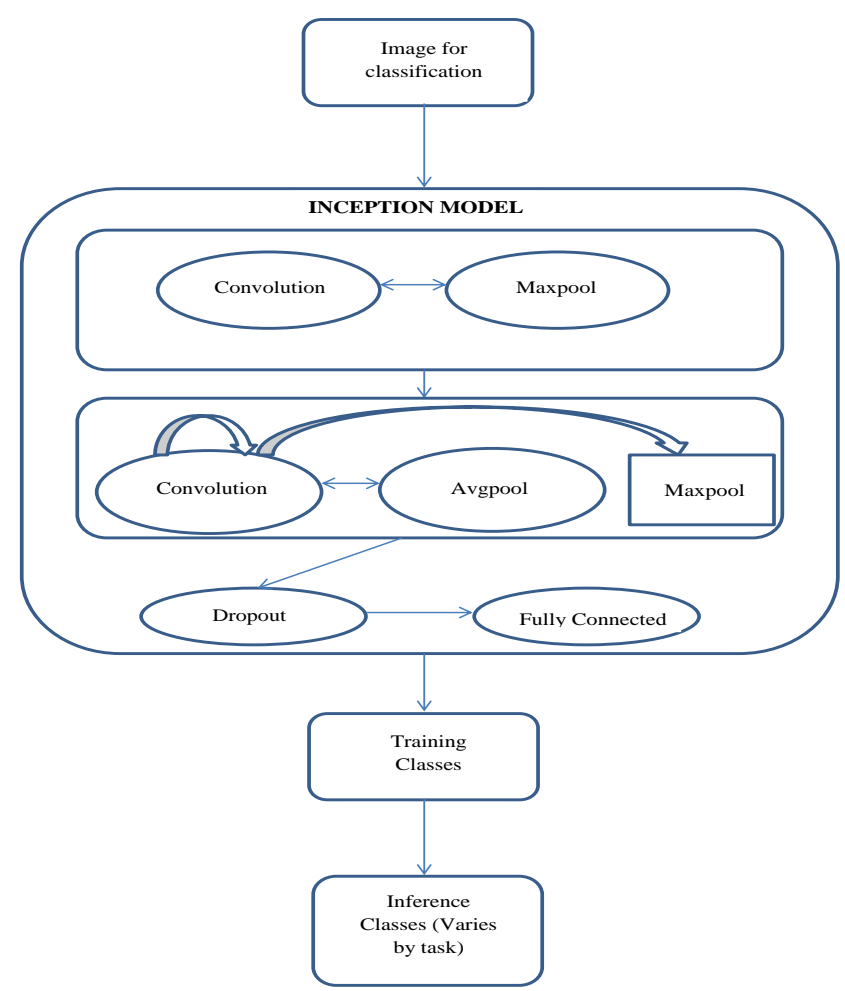

Fig. 5: Architecture of proposed work

Convolution is a step by step process; it extracts diverse features of the input. Each kernel is responsible for producing an output feature [12]. The lower level features of an image like edges, lines, and corners are exacted by lower layers and the higher-level features are extracted by higher layers. So, an input image with height $\mathrm{h}$ and width $\mathrm{w}$ and depth $\mathrm{d}(\mathrm{hXwXd})$, if it is convolved with $M$ kernels, each kernel with height $h 1$, width w1 and depth $d 1$ (h1Xw1Xd1), individually produces $\mathrm{M}$ features.

To make the features obtained from convolution robust against noise, pooling is applied. The resolution of features is reduced by pooling. Pooling can be done via max pooling or average pooling. Fig. 6 depicts pooling.

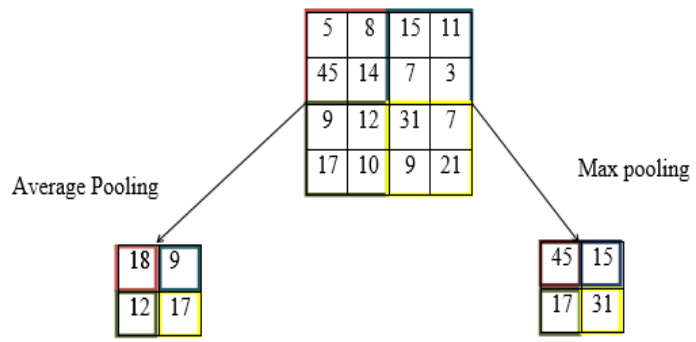

Fig. 6: Average Vs Max Pooling

A standard dataset from ISIC [28] having 13000 images was used for experimentation. The dataset contains two types of cancerous skin images and so they were divided into two folders benign and malignant. Fig. 7 shows the dataset is divided into two folders.

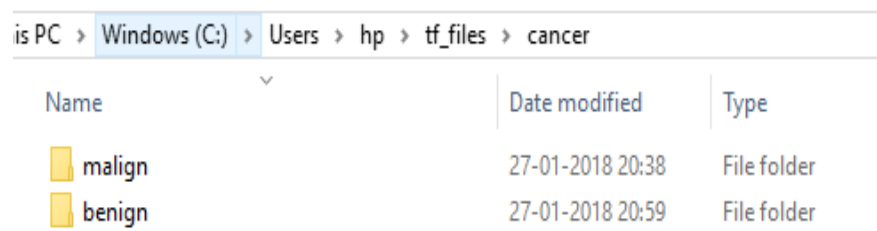

Fig. 7: Dataset Folders

In Fig.8 skin cancer image is taken as input and inception V3 is applied in which convolution, pooling, softmax and fully connected operations are performed. After performing these tasks, it is classified according to various training classes and finally it is labelled as malign and benign cancer.

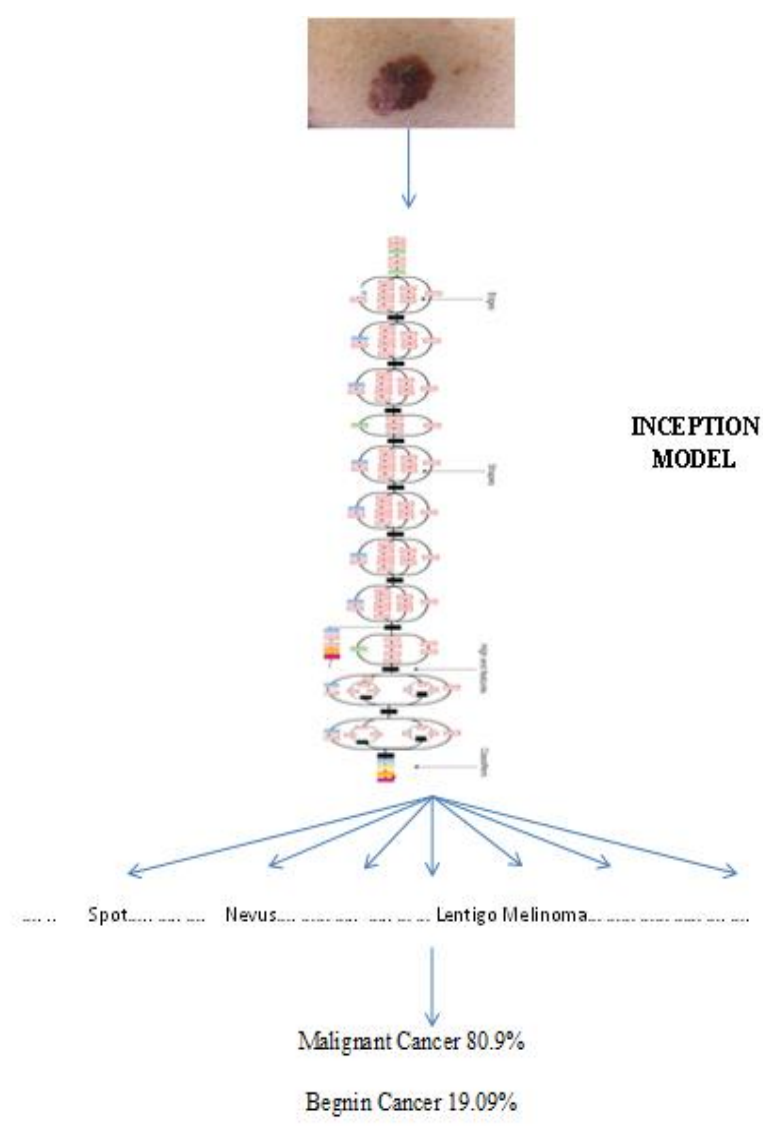

Fig. 8: Skin Cancer Image Classification

The steps in the classification using proposed work are as follows: Algorithm TFclassification \{

Step 1: Begin

Step 2: Build list of images // start training

Step 3: Give path for storing bottle neck values

Step 4: Provide inference to the images //to create bottle neck values

Step 5: Create a folder for all images of bottleneck values

Step 6: Create bottle neck values for every single image

Step 7: Create new softmax layers and fully connected layers // End of training

Step 8: Test the new image

Step 9: End

\section{Experimental results}

The experimental work is done by connecting docker to the virtual box. The neural network is trained to create bottleneck values. Fig.9 shows the generation of bottle neck values. Bottleneck values compress the features to fit in the available space. 


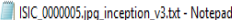

File Edit Format View Help

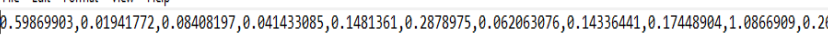
$0.15915062, \theta .07869256,0.2428124, \theta .13715748, \theta .041201144,0.13416025, \theta .24218954,0.49090382, \theta .11478219,0.11248168, \theta$. $386,0.20826456, \theta .24216427,0.2986276,0.842863395, \theta .081876256, \theta .04454358,0.3727851,0.35756788,0.437534,0.84873768, \theta$ $0.5998636,0.05572871,0.14213803,0.017539047,0.07582663,0.06741581,0.2465441,0.4576961,0.50937843,0.26339516,0.257$ $, 0.058833353,0.32684907,0.35561353,0.519577065,0.049285404,0.1609073,0.0,0.9924774,0.20149831,0.0,0.06665495,0.150$ $0.14158301,0.4823318,0.22150299,0.99882644,0.4981131,0.08184368,0.11180212,0.021770539,0.55599225,0.0,0.024016054$ $94,0.35939866,0.010521285,0.0048679,0.6564458,0.045206536,0.18324727,0.23542625,0.8228876,0.5077435,0.17005646,0$. $28628,0.47896472,0.346784066,0.26810184,0.04177554,0.11062724,0.44283837,0.49996927,0.0,0.14101897,0.34545328, \theta .27$

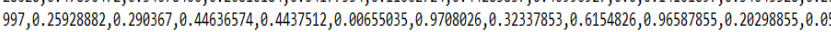
$377637,0.26912177,0.24565233,0.113864124,0.693789,0.13655378,0.22242638,0.3521245,0.24951637,0.4086997,0.42636704$ $0.18518116,0.07071196,1.2034231,0.28778544,0.8357196,0.078944534,0.23706399,0.85995644,0.1699187,1.4150407,0.3415$ $962853,0.065331735,0.007857532, \theta .48764792,1.1030152, \theta .013795568,0.40359783, \theta .10441542, \theta .19696663,0.0408982,0.0148$ $4,0.0843279575,1.7216873,0.7495651, \theta .14080879,0.22138299, \theta .010267896, \theta .84988196, \theta .085929625,0.15696219, \theta .4966189$, $2135,0.011204147,0.13905303,0.010722939,0.046378,0.023836855,0.041009784, \theta .1666894,0.75672144,0.0845598534,0.01349$

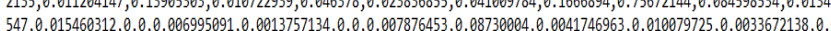

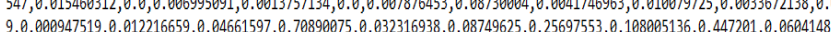

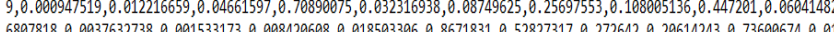
$6807818,0.0037632738,0.061533173,0.068420688,0.018503366,0.8671831,0.52827317,0.272642, \theta .20614243,0.73600674,0.01$

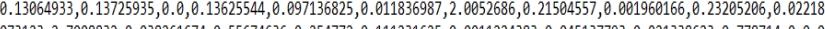
$073123,2.7068832,0.03826164,0.55674636,0.25472,0.111231625,0.0011224383,0.045137793,0.021338023,0.778714,0.0,0$.

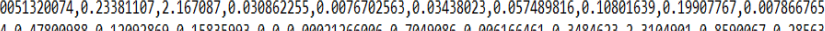

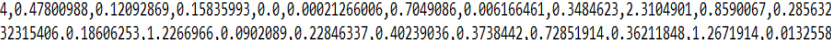

Fig.12 shows the test accuracy vs. no. of iterations graph. The graph shows that the test accuracies are increasing with the increase in number of iterations.

Fig.13 shows the result when a sample test image is taken. As the score of benign is high compared malign, the test image classified as benign.

\section{Conclusion}

Image classification of skin cancer using DCNN with tensor flow and inception V3 models is implemented on CPU version. By using bottleneck values, good accuracy can be achieved even with $\mathrm{CPU}$ version. By increasing the number of iterations, the proposed approach got more than $85 \%$ accuracy.

Fig.10: Fig.11 shows the generation of bottle neck values when the number of iterations is 10 and 100 respectively. It also indicates the accuracies.

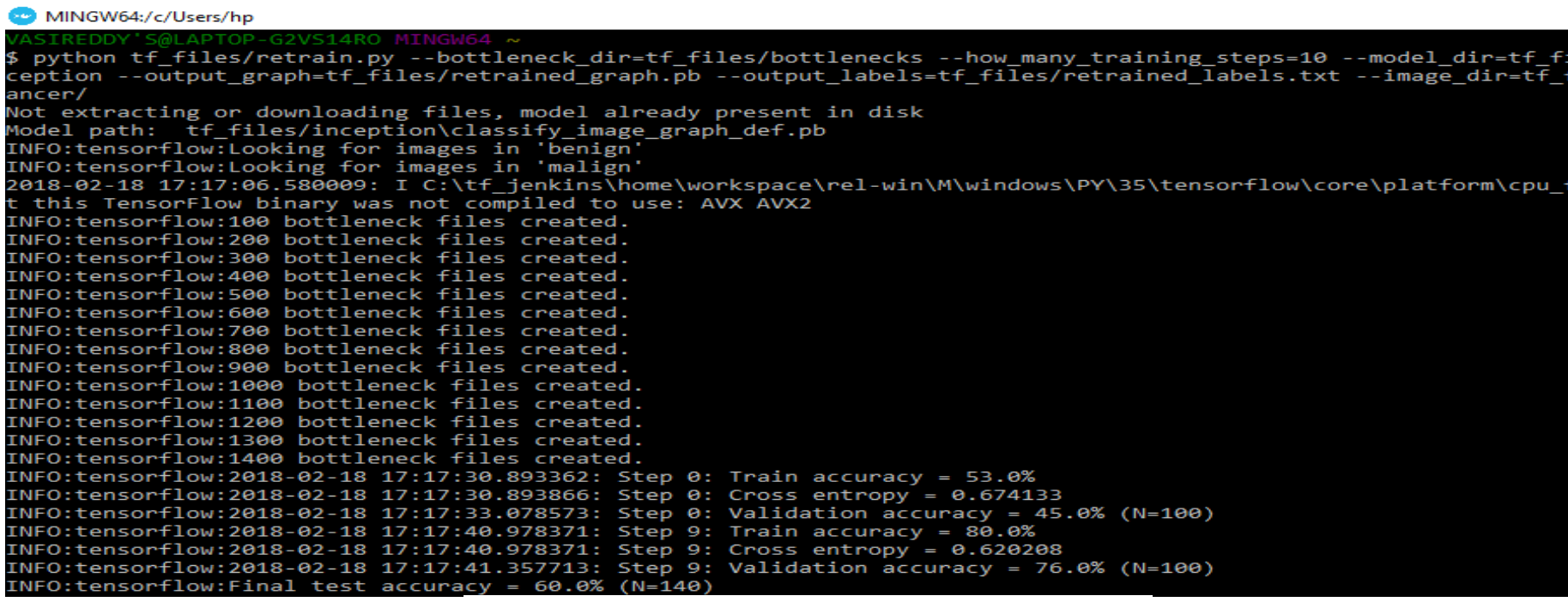

Fig. 10: Creation of bottle neck values for 10 Iterations

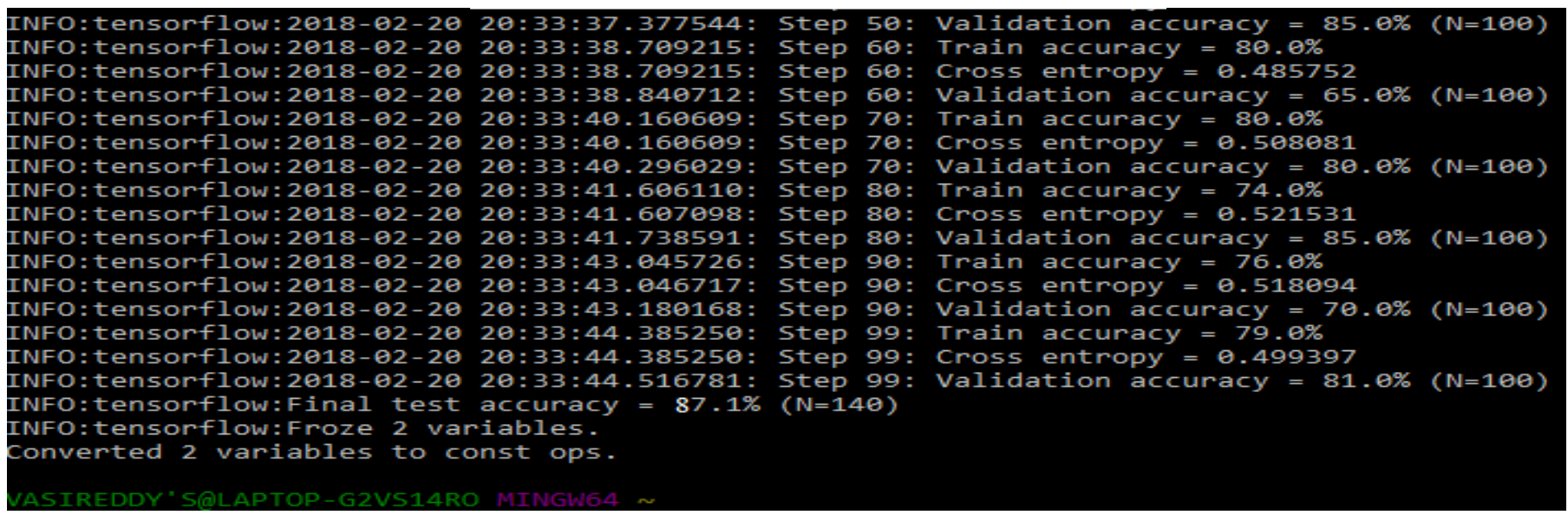

Fig. 11: Creation of bottle neck values for 100 Iterations 


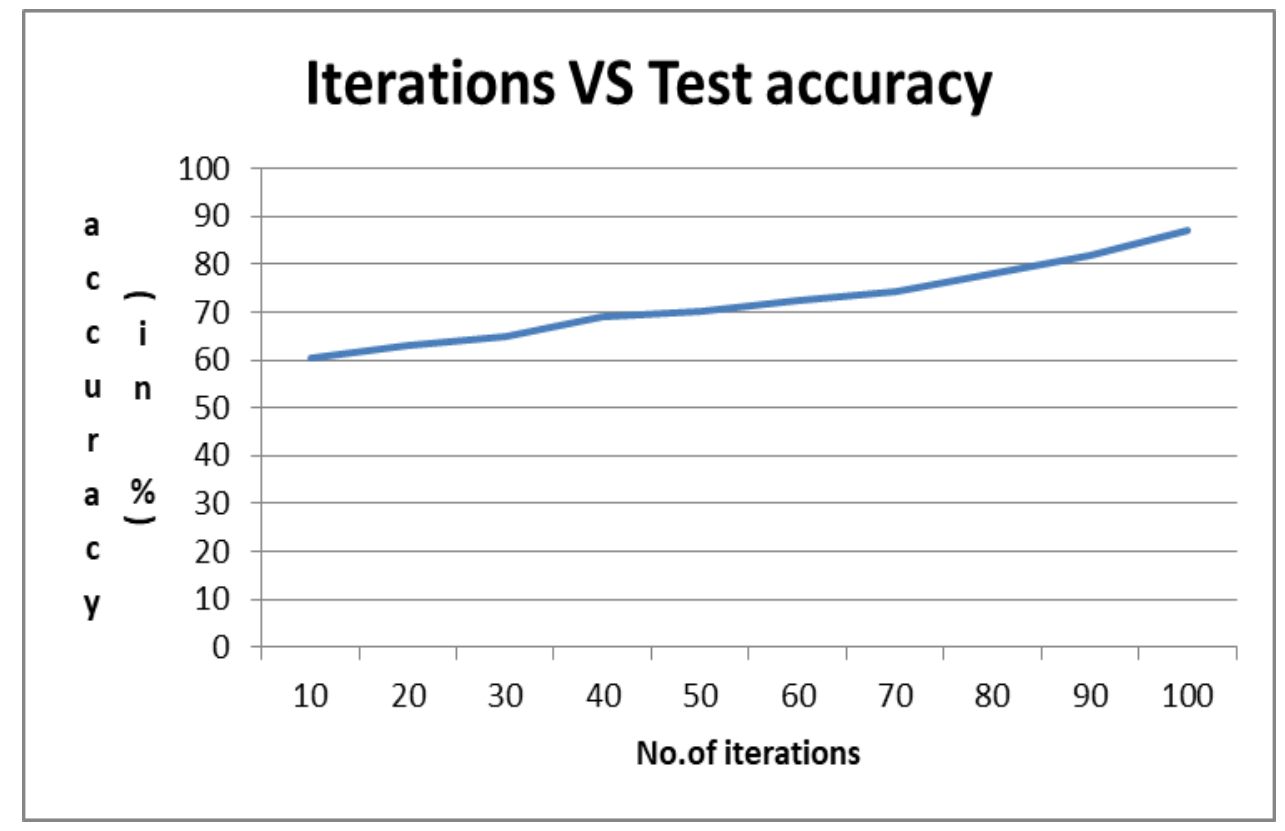

\section{References}

[1] Skin Cancer Facts and Statistics "Available online: https://www.skincancer.org/skin-cancer-information/skin-cancerfacts". Accessed January 20, 2018

[2] M. Fornaciali, M. Carvalho, F. VasquesBittencourt, S. Avila, and E. Valle, "Towards automated melanoma screening: Proper computer vision \& reliable results," arXiv preprint arXiv:1604.04024, 2016.

[3] Melonama and Other Skin Cancers "Available online: https://www.umm.edu/health/medical/ reports/articles/melanoma-and-other-skin-cancers" Accessed: January 20, 2018

[4] Esteva, A., Kuprel, B., Novoa, R. A., Ko, J., Swetter, S. M., Blau, H. M., \&Thrun, S., “ Dermatologist-level classification of skin cancer with deep neural networks" Nature, 542(7639), 115, 2017.

[5] Peng, P., Zhao, X., Pan, X., \& Ye, W, Gas Classification Using Deep Convolutional Neural Networks. Sensors, 18(1), 157,2017

[6] Abadi, M., Barham, P., Chen, J., Chen, Z., Davis, A., Dean, J. and Kudlur, M, "TensorFlow: A System for Large-Scale Machine Learning". In OSDI Vol. 16, pp. 265-283, Nov- 2016.

[7] Szegedy, C., Ioffe, S., Vanhoucke, V., \&Alemi, A. A., "Inception-v4, Inception-ResNet and the Impact of Residual Connections on Learning", In $A A A I$, Vol. 4, p. 12, Feb- 2017.

[8] Lisa Fayed, "Differences between a Malignant and Benign Tumour" "Available online: https://www.verywell.com/what-doesmalignant-and-benign-mean-514240". Accessed December 26, 2017.

[9] Ty Bollinger, "Benign and Malignant Tumours What is the Difference" "Available online: https://thetruthaboutcancer.com/benign-malignant-tumorsdifference/". Accessed January 5, 2018.

[10] Google Cloud Platform, Image classification Transfer learning with Inception V3 “Available online:

https://codelabs.developers.google.com/codelabs/cpb102-txflearning/index.html". Accessed December 20, 2017.

[11] Samer Hijazi, Rishi Kumar, and Chris Rowen Using Convolutional Neural Networks for Image Recognition Cadence IPGroup, White Paper, 2015.

[12] Slaven Bilac," How to classify images with TensorFlow using Google Cloud Machine Learning and Cloud Dataflow" "Available online: https://cloud.google.com/blog/big-data/2016/12/howto-classify-images-with-tensorflow-using-google-cloud-machinelearning-and-cloud-dataflow." Accessed January 12, 2018.

[13] A. Esteva, B. Kuprel, and S. Thrun, "Deep networks for early stage skin disease and skin cancer classification," Stanford, 2015

[14] D. Kumar, M. J. Shafiee, A. Chung, F. Khalvati, M. Haider, and A. Wong, "Discovery radiomics for computed tomography cancer detection," Cornell University Library, 2015.

[15] Hatami, N., Gavet, Y., \&Debayle, J,"Classification of TimeSeries Images Using Deep Convolutional Neural Networks". arXiv preprint arXiv:1710.00886, 2017
[16] Li, Y., \& Shen, L., "Skin lesion analysis towards melanoma detection using deep learning network", arXiv preprint arXiv: 1703.00577,2017

[17] Liao, H, "A deep learning approach to universal skin disease classification", University of Rochester Department of Computer Science, CSC, 2017

[18] Cicero, F. M., Oliveira, A. H. M., Botelho, G. M., \& da Computaçao, C. D. C.," Deep learning and convolutional neural networks in the aid of the classification of melanoma" SIBGRAPI, 2016

[19] Catalina Gómez, Diana Sofia Herrera, "Recognition of skin melanoma through dermoscopic image analysis", Proc. SPIE 10572 13th International Conference on Medical Information Processing and Analysis, 1057211,17- Nov- 2017

[20] Singhal, E., \& Tiwari, S., "Skin cancer detection using artificial neural network", International Journal of Advanced Research in Computer Science, 6(1), 2015

[21] Nilkamal S. Ramteke, Shweta V.Jain."Analysis of Skin Cancer Using Fuzzy and Wavelet Technique - Review \& Proposed New Algorithm". International Journal of Engineering Trends and Technology, VOL. 4(6):2555-2566, Jun- 2013.

[22] Menegola, A., Fornaciali, M., Pires, R., Bittencourt, F. V., Avila, S., \& Valle, E "Knowledge transfer for melanoma screening with deep learning" In Biomedical Imaging (ISBI 2017), IEEE 14th International Symposium on, pp. 297-300, 2017

[23] X. Sun, J Yang, M. Sun, and K. Wang, "A benchmark for automatic visual classification of clinical skin disease images," in ECCV, pp. 206-222, 2016

[24] N. Codella, Q.-B. Nguyen, S. Pankanti, D. Gutman, B. Helba, A. Halpern, and J. Smith, "Deep learning ensembles for melanomarecognitionindermoscopyimages," IBMJResDev, vol. 61 , 2017.

[25] Xia, X., Xu, C., \& Nan, B, “ Inception-v3 for flower classification", In Image, Vision and Computing (ICIVC), 2017 2nd International Conference on ,IEEE, pp. 783-787, June-2017

[26] Nivrito, A. K. M., Wahed, M., \& Bin, R. "Comparative analysis between Inception-v3 and other learning systems using facial expressions detection “,Doctoral dissertation, BRAC University, 2016

[27] ISIC Archive "Available online: https://isicarchive.com/\#images" Accessed December 12, 2017. 